\title{
Supramolecular control of the magnetic anisotropy in two-dimensional high-spin Fe arrays at a metal interface
}

\author{
Pietro Gambardella ${ }^{1,2,3 \star}$, Sebastian Stepanow ${ }^{1,4}$, Alexandre Dmitriev ${ }^{4,5}$, Jan Honolka ${ }^{4}$, \\ Frank M. F. de Groot ${ }^{6}$, Magalí Lingenfelder ${ }^{4}$, Subhra Sen Gupta ${ }^{7}$, D. D. Sarma7 , Peter Bencok ${ }^{8}$, \\ Stefan Stanescu ${ }^{8}$, Sylvain Clair ${ }^{3}$, Stéphane Pons ${ }^{3}$, Nian Lin ${ }^{4}$, Ari P. Seitsonen ${ }^{9}$, Harald Brune ${ }^{3}$, \\ Johannes V. Barth ${ }^{10}$ and Klaus Kern ${ }^{3,4}$
}

\begin{abstract}
Magnetic atoms at surfaces are a rich model system for solid-state magnetic bits exhibiting either classical ${ }^{1,2}$ or quantum $^{3,4}$ behaviour. Individual atoms, however, are difficult to arrange in regular patterns $s^{1-5}$. Moreover, their magnetic properties are dominated by interaction with the substrate, which, as in the case of Kondo systems, often leads to a decrease or quench of their local magnetic moment ${ }^{6,7}$. Here, we show that the supramolecular assembly of Fe and 1,4-benzenedicarboxylic acid molecules on a $\mathrm{Cu}$ surface results in ordered arrays of high-spin mononuclear Fe centres on a $1.5 \mathrm{~nm}$ square grid. Lateral coordination with the molecular ligands yields unsaturated yet stable coordination bonds, which enable chemical modification of the electronic and magnetic properties of the Fe atoms independently from the substrate. The easy magnetization direction of the Fe centres can be switched by oxygen adsorption, thus opening a way to control the magnetic anisotropy in supramolecular layers akin to that used in metallic thin films ${ }^{8-11}$.
\end{abstract}

The control of magnetic anisotropy is a key issue in the development of molecule-metal interfaces for magnetic applications, both at the single-molecule ${ }^{12}$ and extended-film level ${ }^{13}$. In metallic multilayers used as storage media or spin-valve devices at present, tuning of the magnetic anisotropy is achieved either by a careful choice of the overlayer/substrate composition and thickness or by oxidation of the magnetic elements ${ }^{11,14,15}$. Recent studies showed that the magnetization direction of surface-supported paramagnetic molecules can be controlled through exchange coupling with a magnetic film, which provides robust ferromagnetic properties but does not enable each molecule to be switched independently from the substrate or its neighbours ${ }^{16,17}$. Alternatively, theoretical work suggested that the sign of magnetic anisotropy could be reversed in metal-organic complexes by exploiting oxidation processes that affect the hybridization of molecular orbitals with metal states carrying non-zero orbital magnetization ${ }^{18}$.

Here, we investigate supramolecular self-assembly on a non-magnetic $\mathrm{Cu}$ surface as a means to produce two-dimensional
(2D) arrays of regularly spaced Fe spins, the magnitude and magnetic anisotropy of which are manipulated by lateral and axial molecular ligands. Although $\mathrm{Fe}$ atoms adsorbed on $\mathrm{Cu}(100)$ constitute a metallic system owing to the substantial overlap of the $\mathrm{Fe} 3 d$ and $4 s$ states with the $\mathrm{Cu}$ bands, and exhibit a Kondo temperature of about $55 \mathrm{~K}$ (ref. 7), X-ray absorption spectroscopy (XAS) and multiplet calculations shows that $\mathrm{Fe}-\mathrm{Cu}$ hybridization is drastically weakened by square-planar coordination to 1,4-benzenedicarboxylate molecules (hereafter indicated as terephthalate acid or TPA), leading to a high-spin configuration with strongly localized $\mathrm{Fe}^{2+}$ character. X-ray magnetic circular dichroism (XMCD) measurements confirm the presence of sizeable spin moments at the Fe sites, together with a large orbital moment and in-plane magnetic anisotropy that originate from spin-orbitinduced mixing of ground and first excited molecular states. The Fe centres in the supramolecular arrays act as selective adsorption sites for $\mathrm{O}_{2}$, which produces an out-of-plane reorientation of the easy magnetization axis at the $\mathrm{Fe}$ sites. Furthermore, the saturation of the orbital magnetic moments of TPA-coordinated $\mathrm{Fe}$ is found to be strongly anisotropic, revealing an unusual feature of surface-supported magnetic systems.

The co-deposition of transition-metal ions and organic ligands on crystalline surfaces offers the potential to design supramolecular grids with programmable structural and chemical features ${ }^{19,20}$, where the interaction with the substrate is used to stabilize a planar geometry ${ }^{21,22} . \mathrm{Fe}_{x}(\mathrm{TPA})_{y}$ complexes on $\mathrm{Cu}(100)$ constitute a prototypical $2 \mathrm{D}$ hetero-assembled system forming a variety of mono- and bi-nuclear network structures, in which the morphology is determined by the Fe:TPA stoichiometry, substrate symmetry and annealing temperature ${ }^{22-24}$. Figure la shows a scanning tunnelling microscopy image of the square-symmetry array of selfassembled mononuclear $\mathrm{Fe}(\mathrm{TPA})_{4}$ complexes investigated here. Each $\mathrm{Fe}$ atom is coordinated to four TPA molecules through $\mathrm{Fe}$-carboxylate bonds, with the supramolecular $\mathrm{Fe}(\mathrm{TPA})_{4}$ units organized in a $(6 \times 6)$ unit cell with respect to the underlying $\mathrm{Cu}$ lattice (Fig. 1c). Weak hydrogen-bonding interactions between

\footnotetext{
${ }^{1}$ Centre d'Investigacions en Nanociència i Nanotecnologia (ICN-CSIC), UAB Campus, E-08193 Barcelona, Spain, ${ }^{2}$ Institució Catalana de Recerca i Estudis Avançats (ICREA), E-08010 Barcelona, Spain, ${ }^{3}$ Institut de Physique des Nanostructures, Ecole Polytechnique Fédérale de Lausanne (EPFL), CH-1015 Lausanne, Switzerland, ${ }^{4}$ Max-Planck-Institut für Festkörperforschung, D-70569 Stuttgart, Germany, ${ }^{5}$ Department of Applied Physics, Chalmers University of Technology, 41296 Göteborg, Sweden, ${ }^{6}$ Department of Chemistry, Utrecht University, 3584 CA Utrecht, The Netherlands, ${ }^{7}$ Solid State and Structural Chemistry Unit, Indian Institute of Science, Bangalore 560 012, India, ${ }^{8}$ European Synchrotron Radiation Facility, BP 200, F-38043 Grenoble, France, ${ }^{9}$ Institut de Minéralogie et de Physique des Milieux Condensé, Université Pierre et Marie Curie, F-75252 Paris, France, ${ }^{10}$ Physik-Department E20, Technische Universität München, D-85748 Garching, Germany. *e-mail: pietro.gambardella@icrea.es.
} 

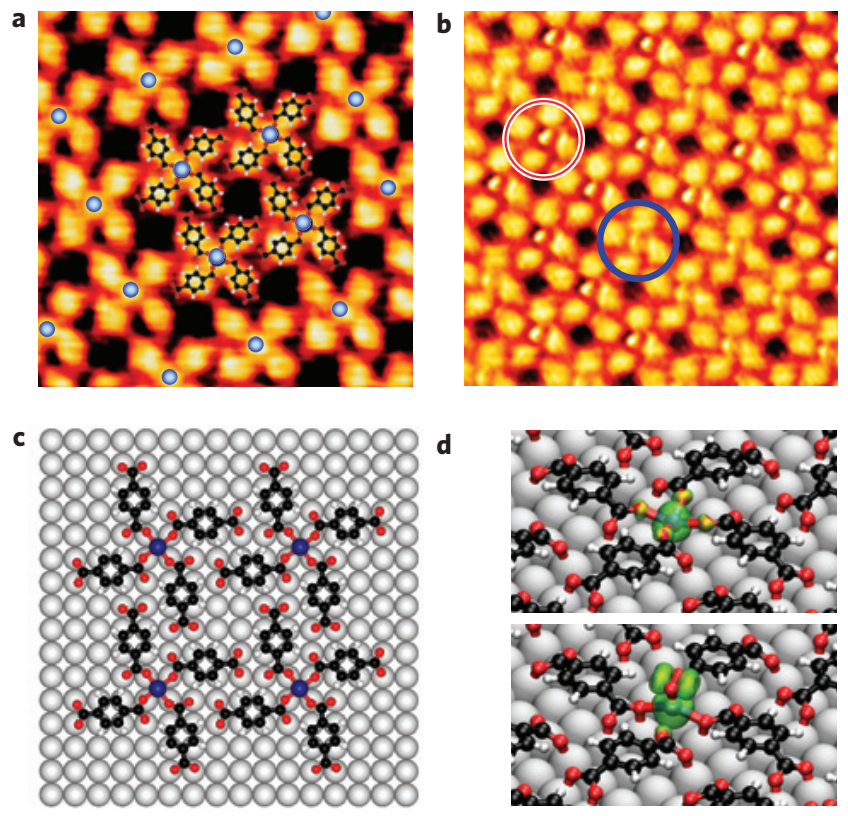

Figure 1 | Planar supramolecular layers of Fe-TPA complexes self-assembled on $\mathbf{C u ( 1 0 0 ) . ~} \mathbf{a}, \mathrm{Fe}(\mathrm{TPA})_{4}$ array; blue dots indicate the position of Fe atoms. Each Fe centre is coordinated to four deprotonated oxygen ligands of the surrounding TPA molecules. STM image size $62 \times 62 \AA^{2}$. b. Selective uptake of $\mathrm{O}_{2}$ by Fe; a change of STM contrast is observed for $\mathrm{O}_{2}-\mathrm{Fe}(T P A)_{4}$ (red circle) at the Fe location compared with $\mathrm{Fe}(\mathrm{TPA})_{4}$ (blue circle). Image size $84 \times 84 \AA^{2}$. c, Top-view model of $\mathrm{Fe}(\mathrm{TPA})_{4}$ coordination and supporting $\mathrm{Cu}(100)$ substrate. d, Relaxed $\mathrm{Fe}(\mathrm{TPA})_{4}$ and $\mathrm{O}_{2}-\mathrm{Fe}(\mathrm{TPA})_{4}$ supramolecular structures obtained by DFT. $\mathrm{Fe}$ (blue), $\mathrm{O}$ (red), $\mathrm{C}$ (black) and $\mathrm{H}$ (white) atoms are shown together with the spin density distribution (green).

the complexes favour long-range order extending over entire terraces of the substrate ${ }^{23}$ (see Supplementary Information, Fig. S1). The resultant superlattice of individual Fe centres has perfect $15 \times 15 \AA^{2}$ periodicity. Despite the four-fold lateral coordination to the carboxylate ligands and the residual interaction with $\mathrm{Cu}, \mathrm{Fe}$ centres are chemically active, forming an array of coordinatively unsaturated sites. On exposure to $\mathrm{O}_{2}$, scanning tunnelling microscopy images indicate that selective adsorption of oxygen takes place close to Fe on-top positions, as demonstrated by the change of contrast in Fig. 1b. The structural properties of both $\mathrm{Fe}(\mathrm{TPA})_{4}$ and $\mathrm{O}_{2}-\mathrm{Fe}(\mathrm{TPA})_{4}$ complexes as well as their interaction with the substrate were further characterized using density functional theory (DFT) calculations, as described in the Methods section. The relaxed supramolecular structures are shown in Fig. 1d together with an isocontour of the spin density distribution; the optimal $\mathrm{O}_{2}-\mathrm{Fe}(\mathrm{TPA})_{4}$ geometry corresponds to $\mathrm{O}_{2}$ chemisorbed on Fe with the $\mathrm{O}-\mathrm{O}$ axis parallel to the surface plane, in agreement with the angular dependence of the oxygen XAS (see Supplementary Information, Fig. S4).

$L$-edge X-ray absorption spectra provide a unique probe of the bonding and magnetic properties of transition-metal ions ${ }^{25}$, with the extra advantage of extreme sensitivity to surface-dilute systems ${ }^{26}$. This is of crucial importance for the present study, as the intensity, lineshape and polarization dependence of dipole-allowed $2 p \rightarrow 3 d$ transitions offer information on the spin state, oxidation state and ligand field of Fe ions in an element-selective way. Figure 2 shows the XAS and XMCD spectra of 0.025 monolayers of Fe deposited on $\mathrm{Cu}(100)$ at room temperature (Fig. 2a), equivalent to the Fe content in the supramolecular arrays, $\mathrm{Fe}$ (TPA) ${ }_{4}$ (Fig. 2b) and $\mathrm{O}_{2}-\mathrm{Fe}$ (TPA) $)_{4}$ (Fig. 2c). The XAS intensity, shown after $\mathrm{Cu}$ background subtraction, was measured at $8 \mathrm{~K}$ with magnetic field
$\mathbf{B}=6 \mathrm{~T}$ applied out-of-plane $\left(\theta=0^{\circ}\right)$ and at grazing incidence $\left(\theta=70^{\circ}\right)$ for parallel $\left(I^{+}\right)$and antiparallel $\left(I^{-}\right)$alignment of the photon helicity with B. More details about the measurements are given in Supplementary Information. The comparison of Fig. $2 \mathrm{a}-\mathrm{c}$ reveals telltale differences in the XAS lineshape: whereas $\mathrm{Fe} / \mathrm{Cu}(100)$ exhibits broad $L_{3}$ and $L_{2}$ peaks typical of a metal, pronounced narrowing of the spectral features and a well-defined multiplet fine structure is observed for the Fe-TPA complexes, similar to the spectra of Fe ions in bulk metal-organic compounds ${ }^{27}$. These changes, related to ligand-induced modifications and localization of the Fe $d$-orbitals, indicate that true coordination bonds have been formed between the Fe centres and carboxylate ligands, with partial decoupling of Fe from the metal substrate. Interestingly, electron localization effects are found to be more pronounced in self-assembled $\mathrm{Fe}(\mathrm{TPA})_{4}$ compared with chemically synthesized $\mathrm{Fe}$-porphyrin molecules evaporated on metal films ${ }^{17}$. We find that the XAS $\left(I^{+}, I^{-}\right)$and the XMCD $\left(I^{+}-I^{-}\right)$spectral shape depend markedly on the orientation of the beam with respect to the molecular arrays. This is in essence a linear dichroism effect ${ }^{25}$ related to the anisotropic spatial distribution of the Fe $d$-electrons in a low-symmetry environment that cannot be observed in bulk-like molecular powder studies ${ }^{27}$, but is resolved here owing to the planar alignment of the complexes. Both the large XAS $L_{3} / L_{2}$ branching ratio and the strong XMCD intensity of $\mathrm{Fe}(\mathrm{TPA})_{4}$ and $\mathrm{O}_{2}-\mathrm{Fe}(\mathrm{TPA})_{4}$ point towards a high-spin Fe configuration for the complexes, against the tendency of the $\mathrm{Cu}$ electrons to reduce the Fe moment through the Kondo interaction ${ }^{7}$.

To gain more insight into the electronic and magnetic properties of the Fe centres, we carried out XAS and XMCD simulations using ligand-field multiplet theory with the inclusion of chargetransfer effects ${ }^{25}$. The molecular environment was modelled by a crystal-field potential defined by the parameters $D q, D s$ and $D t$, as appropriate for $\mathrm{C}_{4 \mathrm{v}}$ symmetry. Covalent mixing of the metal valence $d$-orbitals with the ligand $p$-orbitals was simulated using a valence bond configuration interaction scheme, whereby the ground state of $\mathrm{Fe}$ is taken to be a linear combination of $3 d^{n}$ and $3 d^{n+1} \underline{\mathrm{L}}$ configurations, with $\underline{L}$ indicating a ligand hole. We tested different combinations of $d^{5}, d^{6}$ and $d^{7}$ configurations, corresponding to $\mathrm{Fe}^{3+}, \mathrm{Fe}^{2+}$ and $\mathrm{Fe}^{1+}$, respectively. The simulations show that the ground state of $\mathrm{Fe}$ in both $\mathrm{Fe}(\mathrm{TPA})_{4}$ and $\mathrm{O}_{2}-\mathrm{Fe}(\mathrm{TPA})_{4}$ complexes possesses almost pure $d^{6}$ character, with maximum $14 \%$ and $15 \%$ $d^{7} \underline{L}$ weight, respectively, depending on the hopping parameters assumed in the calculations (see Supplementary Information, Table S1). Within this admixture range, the calculated spectra show only minor differences and are in very good agreement with the experimental data, as shown in Fig. 2d,e. This result has important consequences as (1) it proves that the Fe $d$-orbitals are interacting mainly with the ligands and only weakly perturbed by the metallic substrate (not so for the $s$-states, see Supplementary Information), (2) it shows that $\mathrm{Fe}(\mathrm{TPA})_{4}$ and $\mathrm{O}_{2}-\mathrm{Fe}(\mathrm{TPA})_{4}$ constitute high-spin $\mathrm{Fe}^{2+}$ complexes, which are expected to exhibit zero-field splitting and anisotropic $g$-factors, that is, strong magnetic anisotropy properties in the language of molecular magnetism. In contrast, high-spin $\mathrm{Fe}^{3+}$, with a half-filled $d$-shell, would have only minor orbital contributions to the magnetization and very small magnetic anisotropy.

Consistently with the geometry of the $\mathrm{O}$ ligands, we find that the crystal-field splitting patterns (Fig. $3 \mathrm{c}, \mathrm{d}$ ) are close to square planar and square pyramidal for $\mathrm{Fe}(\mathrm{TPA})_{4}$ and $\mathrm{O}_{2}-\mathrm{Fe}(\mathrm{TPA})_{4}$, respectively, whereas the strong tetragonal distortion that characterizes $\mathrm{Fe}(\mathrm{TPA})_{4}$ reduces significantly in $\mathrm{O}_{2}-\mathrm{Fe}(\mathrm{TPA})_{4}$ owing to the presence of the apical ligand (see Supplementary Information, Table S1). DFT calculations further show that the total number of Fe valence electrons changes from 6.67 to 6.33 following $\mathrm{O}_{2}$ chemisorption, but that the population of the $3 d$-states remains essentially stable (see Supplementary Information, Table S2), in 

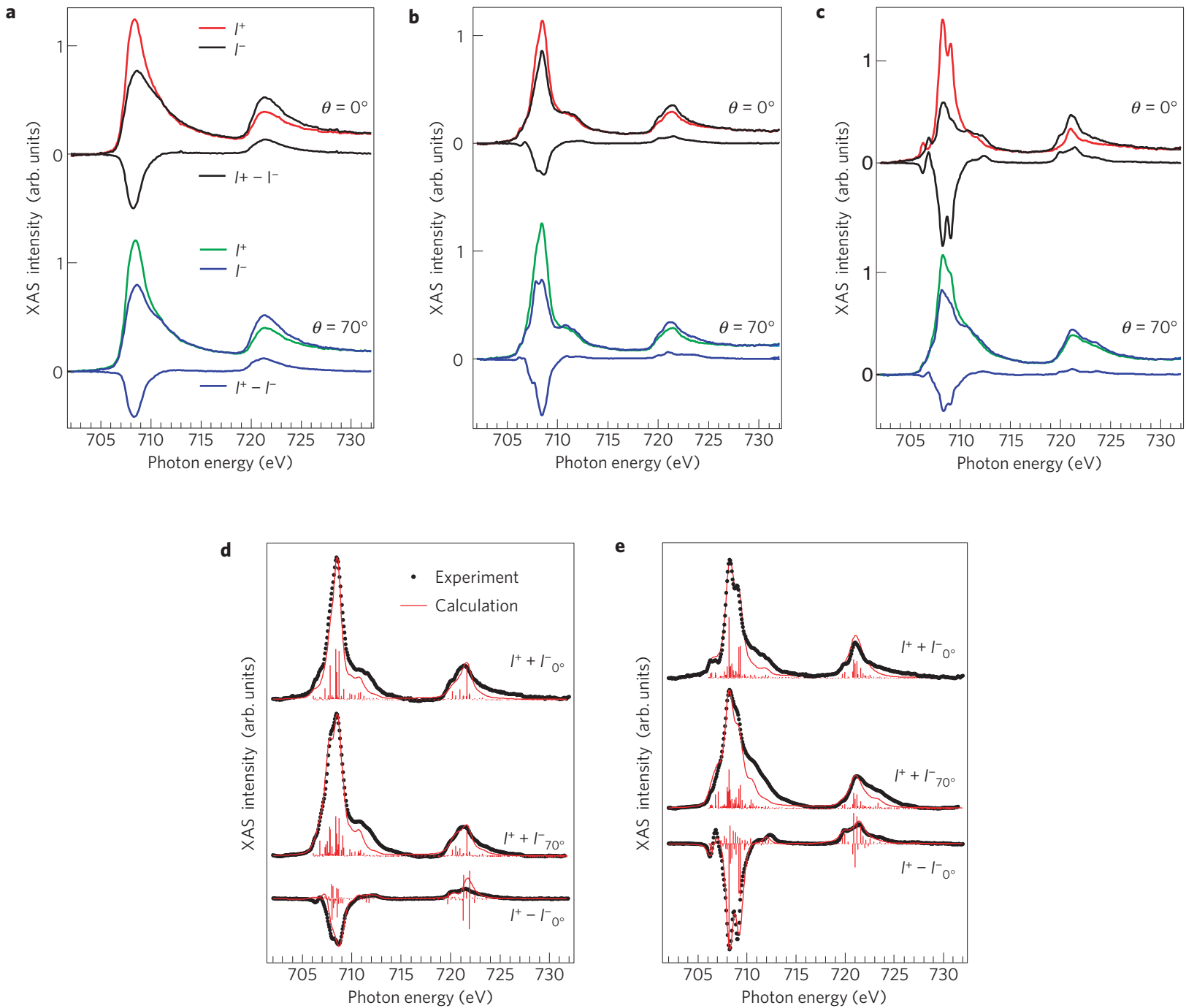

Figure 2 | Circularly polarized X-ray absorption spectra of Fe(TPA $)_{4}, \mathbf{O}_{\mathbf{2}}-\mathrm{Fe}(\mathrm{TPA})_{\mathbf{4}}$ and $\mathrm{Fe} / \mathrm{Cu}(\mathbf{1 0 0})$ measured at the $\mathrm{Fe} \mathbf{L}_{\mathbf{2}, 3}$-edge and calculated multiplet structure. a-c, XAS intensity of 0.025 monolayer $\mathrm{Fe} / \mathrm{Cu}(100)(\mathbf{a}), \mathrm{Fe}(\mathrm{TPA})_{4}(\mathbf{b})$ and $\mathrm{O}_{2}-\mathrm{Fe}(\mathrm{TPA})_{4}(\mathbf{c})$ for parallel $\left(I^{+}\right)$and antiparallel $\left(I^{-}\right)$ orientation of the photon helicity with field-induced magnetization. Spectra were recorded in the electron-yield mode at $T=8 \mathrm{~K}$ and $\mathbf{B}=6 \mathrm{~T}$ at normal $\left(\theta=0^{\circ}\right)$ and grazing incidence $\left(\theta=70^{\circ}\right)$. The XMCD signal $\left(I^{+}-I^{-}\right)$is shown for each orientation. d,e, Calculated XAS and XMCD spectra of Fe $(T P A)_{4}(\mathbf{d})$ and $\mathrm{O}_{2}-\mathrm{Fe}(\mathrm{TPA})_{4}(\mathbf{e})$. See text for details.

agreement with the ligand-field simulations. There are 0.8 excess electrons localized on the $\mathrm{O}_{2}$ ligand, the largest fraction of which is supplied by the $\mathrm{Cu}$ substrate acting as a charge reservoir, a behaviour that is specific to surface-supported metal-organic systems. Notably, the formation of the supramolecular complexes is accompanied by a substantial increase of the Fe-substrate distance, calculated as $2.32 \AA$ for individual $\mathrm{Fe}$ atoms on $\mathrm{Cu}(100)$, $2.71 \AA$ in $\mathrm{Fe}(\mathrm{TPA})_{4}$ and $3.32 \AA$ in $\mathrm{O}_{2}-\mathrm{Fe}(\mathrm{TPA})_{4}$, showing together with XAS how the $\mathrm{Fe}-\mathrm{Cu}$ metal interactions progressively weaken with increasing number of ligands. A more detailed discussion of the crystal-field and DFT electronic-structure results is reported in Supplementary Information.

Confirmation of the strong magnetic anisotropy behaviour of $\mathrm{Fe}$ in the supramolecular complexes was obtained by angle-dependent XMCD measurements, exploiting the XMCD proportionality to the projection of the Fe magnetic moment on the X-ray incidence direction. The relative magnitude of the XMCD spectra at $\theta=0^{\circ}$ and $70^{\circ}$ in Fig. 2 indicates that, whereas $\mathrm{Fe} / \mathrm{Cu}(100)$ has weak out-of-plane anisotropy, the $\mathrm{Fe}(\mathrm{TPA})_{4}$ easy axis lies in-plane. $\mathrm{O}_{2}$ adsorption at the Fe sites, however, further drives an abrupt magnetic anisotropy reorientation transition, rotating the Fe easy axis out-of-plane. The field dependence of the XMCD signal in Fig. 3a,b underlines the strong change in magnetic anisotropy induced by $\mathrm{O}_{2}$. This effect is comparable to that reported for ultrathin metal films ${ }^{9-11}$, but opposite in sign with respect to oxygen-dosed Fe layers on $\mathrm{Cu}(100)$ (ref. 11). With respect to bulk molecular crystals, the planar and open coordination structure of the self-assembled Fe array makes such a system extremely sensitive to chemisorption, providing straightforward control of the preferred Fe spin orientation. Through the analysis of the XAS and XMCD spectra, we can further identify the cause for the easy-axis switch in the metal-organic complexes, revealing a different mechanism from the one proposed by Atodiresei and co-workers ${ }^{18}$ based on the control of the metal oxidation state. Our calculations show that only the ligand field is affected by $\mathrm{O}_{2}$ adsorption, whereas the formal $\mathrm{Fe}$ oxidation does not change. As demonstrated by the crystal-field level schemes in Fig. $3 c$,d, the axial $\mathrm{O}_{2}$ ligand induces a change of the $\mathrm{Fe}$ ground state from $A_{1 g}$ to $E_{g}$. This effect can be understood also in an intuitive way, as the on-top $\mathrm{O}_{2}$ molecule binds axially to the $d_{z^{2}}$ Fe orbital, thus effectively pushing up the antibonding $\mathrm{A}_{\lg }$ state (that is, the term with $d_{z^{2}}$-like symmetry) relative to 

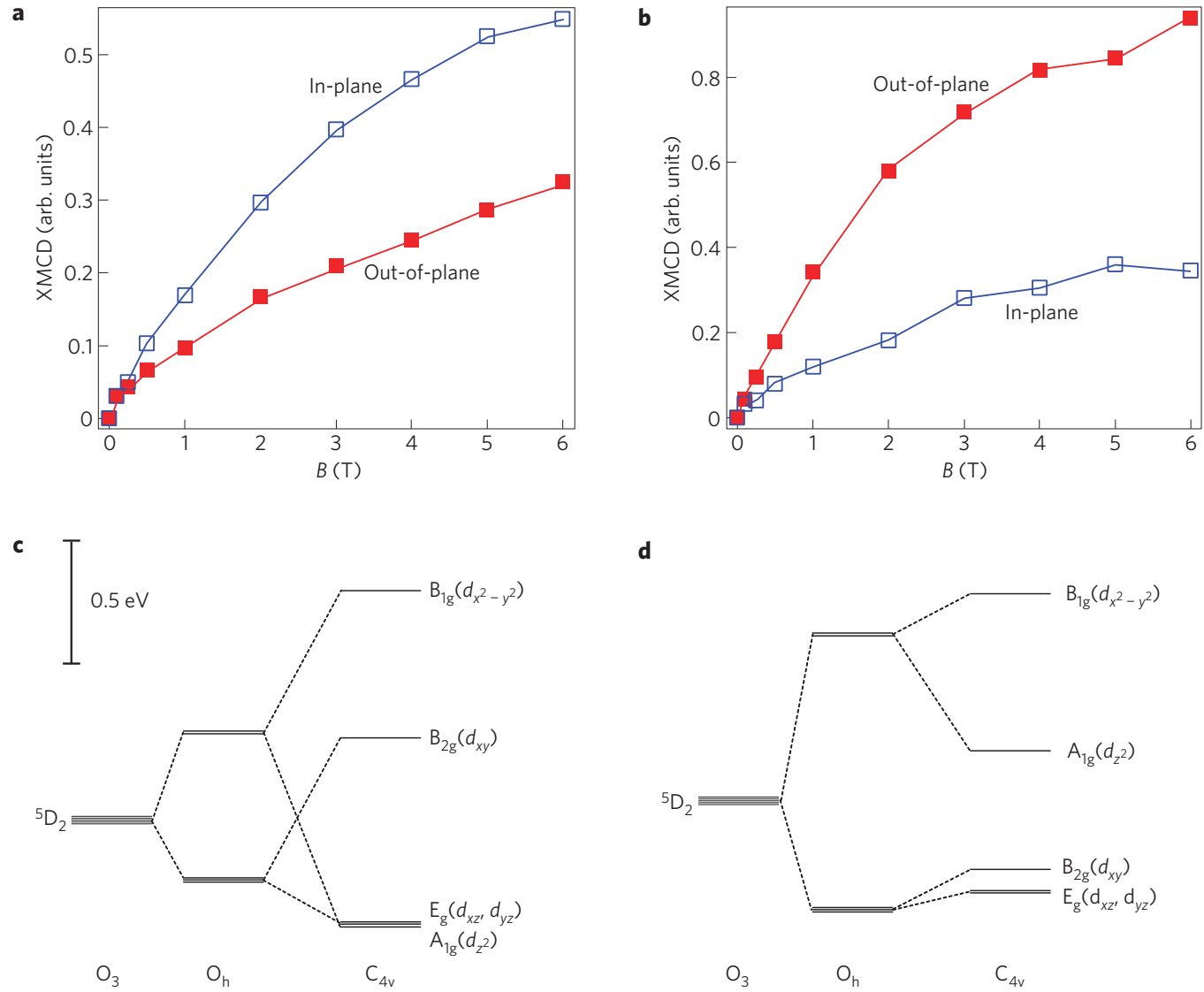

d

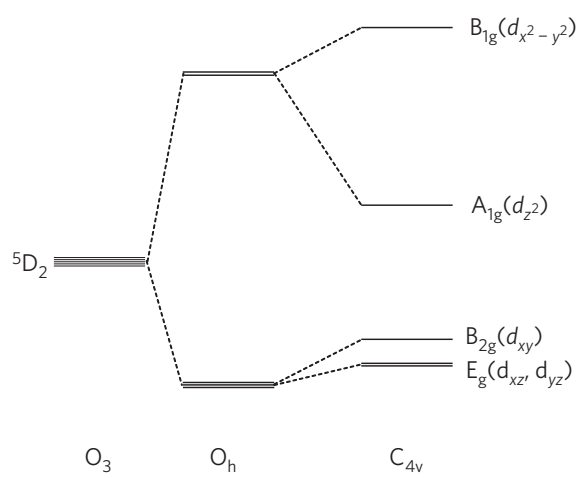

Figure 3 | Element-selective magnetization curves of the Fe centres and crystal-field diagrams. a,b, $\mathrm{Fe}(\mathrm{TPA})_{4}(\mathbf{a})$ and $\mathrm{O}_{2}-\mathrm{Fe}(\mathrm{TPA})_{4}(\mathbf{b})$ magnetization measured at $T=8 \mathrm{~K}$ with the field applied out-of-plane $\left(\theta=0^{\circ}\right.$, filled symbols $)$ and close to the in-plane direction $\left(\theta=70^{\circ}\right.$, open symbols). The data points represent the Fe XMCD intensity integrated over the $L_{3}$-edge and normalized by the corresponding $L_{3}$-integrated XAS intensity. c,d, $C$ rystal-field diagram of $\mathrm{Fe}(T P A)_{4}(\mathbf{c})$ and $\mathrm{O}_{2}-\mathrm{Fe}(\mathrm{TPA})_{4}$ (d) derived from the XAS multiplet simulations.

the $\mathrm{E}_{\mathrm{g}}$ term in $\mathrm{O}_{2}-\mathrm{Fe}(\mathrm{TPA})_{4}$. The change of ground state has important consequences for the magnetic properties: before taking the spin-orbit perturbation on the crystal-field levels into account, the orbital angular momentum is quenched for an $A_{1 g}$ term. In $\mathrm{C}_{4 \mathrm{v}}$ symmetry, on the other hand, the $\mathrm{E}_{\mathrm{g}}$ term is an orbitally degenerate doublet with non-zero orbital moment pointing along the principal symmetry direction. The tendency of $\mathrm{O}_{2}-\mathrm{Fe}(\mathrm{TPA})_{4}$ to magnetize out-of-plane, together with its stronger magnetic anisotropy compared with $\mathrm{Fe}(\mathrm{TPA})_{4}$, can be traced back to its unperturbed crystal-field configuration and non-zero first-order orbital moment perpendicular to the surface plane. In $\mathrm{Fe}(\mathrm{TPA})_{4}$, the orbital moment arises as a second-order perturbation effect as spin-orbit coupling admixes the nearly degenerate $A_{1 g}$ and $\mathrm{E}_{\mathrm{g}}$ states $^{28}$. XMCD sum-rule measurements reflect the difference between the two compounds, showing that $\mathrm{O}_{2}-\mathrm{Fe}(\mathrm{TPA})_{4}$ has $30 \%$ higher orbital magnetic moment compared with $\mathrm{Fe}(\mathrm{TPA})_{4}$, namely $0.55 \pm 0.07$ and $0.42 \pm 0.06 \mu_{\mathrm{B}}$ at $T=8 \mathrm{~K}$, when $\mathbf{B}=6 \mathrm{~T}$ is applied out-of-plane and in-plane, that is, along the respective easy magnetization direction. Note that the orbital magnetic moment measured for $\mathrm{Fe} / \mathrm{Cu}(100)$ is much smaller, $0.18 \pm 0.03 \mu_{\mathrm{B}}$. A complete discussion of the sum-rule analysis is reported in Supplementary Information.

The ligand-field calculations yield zero-field splitting of about -3 and $+5 \mathrm{meV}$ for $\mathrm{Fe}(\mathrm{TPA})_{4}$ and $\mathrm{O}_{2}-\mathrm{Fe}(\mathrm{TPA})_{4}$, respectively. However, we caution that these parameters may be overestimated by as much as a factor of two, as are the ligand-field predictions for the orbital moments, owing to covalency effects that are not treated in the ligand-field approach ${ }^{28}$. Our data disclose an extra unusual effect for surface magnetic systems, namely the strong anisotropy of the saturation magnetic moments of Fe. This effect is borne out both by the XMCD curves in Fig. 3 and the ligand-field calculations, which reveal a large and highly anisotropic orbital magnetization due to the predominant influence of the low-symmetry ligand field over the Fe-metal interaction, and confirmed by the different XMCD multiplet structure observed at $\theta=70^{\circ}$ relative to $0^{\circ}$ (see Supplementary Information, Fig. S2). Finally, we note that no sign of magnetic coupling was detected between the Fe centres. Future investigations may address this issue using different kinds of molecular ligand to adjust the Fe-Fe spacing and spin-dependent electron correlation.

The spin behaviour of individual atoms in purely metallic and molecular environments has been intensively studied in recent years, leading to the discovery of unusual magnetic ${ }^{1-4,12,17}$ and electron transport properties ${ }^{29,30}$. The capability to fabricate $2 \mathrm{D}$ arrays of monodisperse spin centres with nanometre spacing, and to understand and control their magnetic properties at the interface with a metal substrate, constitutes a basic step towards the exploitation of single-spin phenomena in heterogeneous devices based on either a mono- or multilayer geometry.

\section{Methods}

Experiments were carried out at the ID08 beamline of the European Synchrotron Radiation Facility in Grenoble. $\mathrm{Fe}(\mathrm{TPA})_{4}$ and $\mathrm{O}_{2}-\mathrm{Fe}(\mathrm{TPA})_{4}$ layers were prepared by sequential deposition of TPA and Fe on a clean $\mathrm{Cu}(100)$ surface and characterized in situ by STM and low-energy electron diffraction before the XAS measurements. Ultrahigh-vacuum conditions were maintained throughout the structural and XAS characterization measurements. For further details about the sample preparation, multiplet calculations and XMCD analysis, see Supplementary Information. The DFT calculations were carried out using the generalized gradient approximation as the exchange-correlation functional of the Kohn-Sham equations using the 
Vienna Ab-initio Simulation Package code (http://cms.mpi.univie.ac.at/vasp/). The electronic wavefunctions were expanded in a plane-wave basis set, up to a cutoff energy of $37 \mathrm{Ryd}$, and the core-valence interaction was modelled with the projected augmented-wave method. Three substrate layers (first fully relaxed with adsorbate layer, second only laterally) together with almost $20 \AA$ of vacuum between the two surfaces of the slab were used. A $(2 \times 2)$ grid of Monkhorst-Pack $k$-points in the first Brillouin zone was used to approximate the integration over the reciprocal space during the atomic relaxation, and a $(4 \times 4)$ grid was used for the analysis.

Received 17 July 2008; accepted 22 December 2008; published online 1 February 2009

\section{References}

1. Gambardella, P. et al. Giant magnetic anisotropy of single cobalt atoms and nanoparticles. Science 300, 1130-1133 (2003).

2. Meier, F., Zhou, L., Wiebe, J. \& Wiesendanger, R. Revealing magnetic interactions from single-atom magnetization curves. Science 320, 82-86 (2008).

3. Heinrich, A. J., Gupta, J. A., Lutz, C. P. \& Eigler, D. M. Single-atom spin-flip spectroscopy. Science 306, 466-469 (2004).

4. Hirjibehedin, C. F. et al. Large magnetic anisotropy of a single atomic spin embedded in a surface molecular network. Science 317, 1199-1203 (2007).

5. Eigler, D. M. \& Schweitzer, E. K. Positioning single atoms with a scanning electron microscope. Nature 344, 524-526 (1990).

6. Nagaoka, T., Jamneala, T., Grobis, M. \& Crommie, M. F. Temperature dependence of a single Kondo impurity. Phys. Rev. Lett. 88, 077205 (2002).

7. Wahl, P. et al. Kondo effect of molecular complexes at surfaces: Ligand control of the local spin coupling. Phys. Rev. Lett. 95, 166601 (2005).

8. Monso, S. et al. Crossover from in-plane to perpendicular anisotropy in $\mathrm{Pt} / \mathrm{CoFe} / \mathrm{AlOx}$ sandwiches as a function of $\mathrm{Al}$ oxidation: A very accurate control of the oxidation of tunnel barriers. Appl. Phys. Lett. 80, 4157-4159 (2002).

9. Hong, J., Wu, R. Q., Lindner, J., Kosubek, E. \& Baberschke, K. Manipulation of spin reorientation transition by oxygen surfactant growth: A combined theoretical and experimental approach. Phys. Rev. Lett. 92, 147202 (2004).

10. Sander, D. et al. Reversible $\mathrm{H}$-induced switching of the magnetic easy axis in Ni/Cu(001) thin films. Phys. Rev. Lett. 93, 247203 (2004).

11. Peterka, D., Enders, A., Haas, G. \& Kern, K. Adsorbate and thermally induced spin reorientation transition in low-temperature-grown $\mathrm{Fe} / \mathrm{Cu}(001)$. Phys. Rev. B 66, 104411 (2002).

12. Bogani, L. \& Wernsdorfer, W. Molecular spintronics using single-molecule magnets. Nature Mater. 7, 179-186 (2008).

13. Naber, W. J. M., Faez, S. \& van der Wiel, W. G. Organic spintronics. J. Phys. D 40, R205-R228 (2007).

14. Parkin, S. S. P. et al. Magnetically engineered spintronic sensors and memory. Proc. IEEE 91, 661-680 (2003).

15. Fukuzawa, H. et al. Specular spin-valve films with an FeCo nano-oxide layer by ion-assisted oxidation. J. Appl. Phys. 91, 6684-6690 (2002).
16. Scheybal, A. et al. Induced magnetic ordering in a molecular monolayer. Chem. Phys. Lett. 411, 214-220 (2005).

17. Wende, H. et al. Substrate-induced magnetic ordering and switching of iron porphyrin molecules. Nature Mater. 6, 516-520 (2007).

18. Atodiresei, N. et al. Controlling the magnetization direction in molecules via their oxidation state. Phys. Rev. Lett. 100, 117207 (2008).

19. Ruben, M., Rojo, J., Romero-Salguero, F. J., Uppadine, L. H. \& Lehn, J.-M. Grid-type metal ion architectures: Functional metallosupramolecular arrays. Angew. Chem. Int. Ed. 43, 3644-3662 (2004).

20. Stepanow, S. et al. Steering molecular organization and host-guest interactions using two-dimensional nanoporous coordination systems. Nature Mater. 3 , 229-233 (2004).

21. Semenov, A. et al. Controlled arrangement of supramolecular metal coordination arrays on surfaces. Angew. Chem. Int. Ed. 38, 2547-2550 (1999).

22. Barth, J. V., Costantini, G. \& Kern, K. Engineering atomic and molecular nanostructures at surfaces. Nature 437, 671-679 (2005).

23. Lingenfelder, M. A. et al. Towards surface-supported supramolecular architectures: Tailored coordination assembly of 1,4-benzenedicarboxylate and Fe on $\mathrm{Cu}(100)$. Chem. Eur. J. 10, 1913-1919 (2004).

24. Stepanow, S., Lin, N. \& Barth, J. V. Modular assembly of low-dimensional coordination architectures on metal surfaces. J. Phys. Condens. Matter 20, 184002 (2008).

25. de Groot, F. \& Kotani, A. Core Level Spectroscopy of Solids (CRC Press, 2008).

26. Gambardella, P. et al. Localized magnetic states of Fe, Co, and Ni impurities on alkali metal films. Phys. Rev. Lett. 88, 047202 (2002).

27. Hocking, R. K. et al. Fe L-edge X-ray absorption spectroscopy of low-spin heme relative to non-heme Fe complexes: Delocalization of Fe d-electrons into the porphyrin ligand. J. Am. Chem. Soc. 129, 113-125 (2007).

28. Figgis, B. N. \& Hitchman, M. A. Ligand Field Theory and Its Applications (Wiley-VCH, 2000).

29. Rocha, A. R. et al. Towards molecular spintronics. Nature Mater. 4, 335-339 (2005).

30. Timm, C. \& Elste, F. Spin amplification, reading, and writing in transport through anisotropic magnetic molecules. Phys. Rev. B 73, 235304 (2006).

\section{Acknowledgements}

We acknowledge the ESRF for provision of beam time. Partial financial support was received through the EUROCORES 05-SONS-FP-009 SANMAG project of the European Science Foundation. P.G. and S.S. acknowledge financial support from the Spanish Ministerio de Educación y Ciencia (SYNSPIN-MAT2007-62341).

\section{Additional information}

Supplementary Information accompanies this paper on www.nature.com/naturematerials. Reprints and permissions information is available online at http://npg.nature.com/ reprintsandpermissions. Correspondence and requests for materials should be addressed to P.G. 